\title{
The role of antimalarials in lupus nephritis: a review
}

Senq-J Lee ${ }^{1 *}$, Earl D Silverman', Joanne M Bargman²

From 2011 Pediatric Rheumatology Symposium sponsored by the American College of Rheumatology Miami, FL, USA. 2-5 June 2011

\section{Purpose}

Systemic lupus erythematosus (SLE) is a chronic multisystem autoimmune disease affecting various organs, with lupus nephritis being one of the most important and common serious manifestations. Antimalarials (AM) are one of the many immunomodifying medications

\begin{tabular}{|c|c|c|c|c|}
\hline Authors & Year & No.pts & Study & Outcomes of AM use in SLE \\
\hline $\begin{array}{l}\text { Fesaler et al. } \\
\text { LUMINA [38] }\end{array}$ & 2005 & $\begin{array}{l}518(291 \mathrm{HCQ} \\
\text { users, } 227 \text { non- } \\
\text { users) }\end{array}$ & $\begin{array}{l}\text { Longitudinal } \\
\text { cohort }\end{array}$ & $\begin{array}{l}\text { Reduction in accrual of new disease damage (HR 0.6S; } 9 \% \% \mathrm{Cl} \\
0.53-0.93, p=0.014)\end{array}$ \\
\hline $\begin{array}{l}\text { Ruiz-lrastorza et } \\
\text { al[42] }\end{array}$ & 2006 & $\begin{array}{l}232 \text { (150 AM } \\
\text { users, } 82 \text { non- } \\
\text { users) }\end{array}$ & $\begin{array}{l}\text { Prospective } \\
\text { cohort }\end{array}$ & $\begin{array}{l}\text { Increased survival (cumulative } 15 y \text { r survival } 0.95 \text { AM va. } 0.6 \text { non- } \\
\text { AM, p.0.001; HR } 0.14 \text { (95\%Cl 0.04-0.48)) }\end{array}$ \\
\hline $\begin{array}{l}\text { Calvo-Alen et al } \\
\text { LUMINA [43] }\end{array}$ & 2006 & $\begin{array}{l}32 \text { SLE pts with } \\
\text { osteonecrosis va. } \\
59 \text { matched } \\
\text { controls }\end{array}$ & $\begin{array}{l}\text { Nested case- } \\
\text { control }\end{array}$ & Possible protection against osteoporosis \\
\hline Barber et al[44] & 2006 & $\begin{array}{l}35 \text { patients with } \\
\text { UN (16 sustained } \\
\text { remission vs. } 19 \\
\text { controls) }\end{array}$ & $\begin{array}{l}\text { Retrospective } \\
\text { cohort }\end{array}$ & Improved sustained remission rates: \\
\hline $\begin{array}{l}\text { Wosniacka et } \\
\text { al[14] }\end{array}$ & 2006 & $\begin{array}{l}25 \text { SLE pts va. } 25 \\
\text { control pts }\end{array}$ & Cohort & Improved reduction in SLAM score: \\
\hline $\begin{array}{l}\text { Coatedoat:- } \\
\text { Chalumeau et } \\
\text { al[45] }\end{array}$ & 2006 & $\begin{array}{l}143 \text { SLE pts (120 } \\
\text { inactive disease va. } \\
23 \text { active disease) }\end{array}$ & $\begin{array}{l}\text { Longitudinal } \\
\text { cohort }\end{array}$ & $\begin{array}{l}\text { Lower } \mathrm{HCQ} \text { levels in patients with flares }(\mathrm{OR} 0.4,95 \% \mathrm{Cl} 0.18 \text {. } \\
9.55, p=0.01)\end{array}$ \\
\hline $\begin{array}{l}\text { Alarcon GS et al. } \\
\text { LUMINA [37] }\end{array}$ & 2007 & $\begin{array}{l}608 \text { SLE pts: } 547 \\
\text { live (controls), } 61 \\
\text { deaths (cases) }\end{array}$ & Case-control & Increased survival (OR 0.128, $95 \% \mathrm{Cl} 0.054-0.3)$ \\
\hline $\begin{array}{l}\text { Ruiz-lrastorza et } \\
\text { al[46] }\end{array}$ & 2007 & $\begin{array}{l}235 \text { SLE pts (156 } \\
\text { AM users va: } 79 \\
\text { non-users) }\end{array}$ & $\begin{array}{l}\text { Observational } \\
\text { prospective } \\
\text { cohort }\end{array}$ & $\begin{array}{l}\text { Protective against neoplasia ( } 1.3 \% \text { va. } 13 \%, \text { pc0.001; cumulative } \\
\text { cancer-free survival OR } 0.98 \text { va. } 0.73, \text { p< } 0.001 \text { ) }\end{array}$ \\
\hline Siso et al [47] & 2008 & $\begin{array}{l}206 \text { pts with LN } \\
\text { (56 on } \mathrm{HCQ} \text { prev, } \\
150 \text { non-HCQ) }\end{array}$ & Cohort & $\begin{array}{l}\text { Procective against infection ( } 11 \% \text { va. } 29 \%, p=0.006 \text { ) } \\
\text { Increased survival ( } 2 \% \text { va. } 13 \%, p=0.029)\end{array}$ \\
\hline $\begin{array}{l}\text { Ruiz-lrastorza et } \\
\text { al [48] }\end{array}$ & 2009 & $\begin{array}{l}249 \text { SLE pts: } 83 \text { pts } \\
\text { with infections } \\
\text { (cases) vs. } 166 \text { no } \\
\text { infections } \\
\text { (controls) }\end{array}$ & $\begin{array}{l}\text { Nested case- } \\
\text { control }\end{array}$ & Protective against infection (OR $0.06,95 \% \mathrm{Cl} 0.02-0.18$ ) \\
\hline Shinjo et al [49] & 2009 & $\begin{array}{l}57 \text { SLE pts } 265 \text { vo: } \\
43 \text { disease } \\
\text { remission, } 14 \\
\text { disease activity }\end{array}$ & $\begin{array}{l}\text { Retrospective } \\
\text { cohort }\end{array}$ & $\begin{array}{l}\text { Disease remizsion stronslv associated to AM use (OR 12.9, } \\
95 \% \text { Ci } 2.9-58.1 \text { ) }\end{array}$ \\
\hline $\begin{array}{l}\text { Pons-Estel et al. } \\
\text { LUMINA [50] }\end{array}$ & 2010 & $S S 0$ pts & $\begin{array}{l}\text { Longitudinal } \\
\text { observational } \\
\text { cohort }\end{array}$ & $\begin{array}{l}\text { Possible delayed onset of integument damage (HR } 0.23,95 \% \mathrm{Cl} \\
0.12-0.47 \text { ) }\end{array}$ \\
\hline $\begin{array}{l}\text { Shinjo et al. } \\
\text { GLADEL [40] }\end{array}$ & 2010 & $\begin{array}{c}1480 \text { pts (1141 AMA } \\
\text { users vs. } 339 \text { non- } \\
\text { users) }\end{array}$ & $\begin{array}{l}\text { Longitudinal } \\
\text { cohort }\end{array}$ & $\begin{array}{l}\text { Increased survival ( } 4.4 \% \text { va. } 11.5 \%, p<0.001 ; \mathrm{HR} 0.62,05 \% \mathrm{Cl} \\
0.39-0.99)\end{array}$ \\
\hline
\end{tabular}

Figure 1 Overview of effects of antimalarials on lupus disease and activity - articles from 2005 to 2010. LN=lupus nephritis.

${ }^{1}$ The Hospital for Sick Children, Toronto, ON, Canada

Full list of author information is available at the end of the article

(C) 2012 Lee et al; licensee BioMed Central Ltd. This is an Open Access article distributed under the terms of the Creative Commons 


\begin{tabular}{|c|c|c|c|c|}
\hline Authors & Year & No.pts & Study & Outcomes of AM use in SLE \\
\hline $\begin{array}{l}\text { Ruiz-lrastorza et } \\
\text { al[42] }\end{array}$ & 2006 & $\begin{array}{l}232 \text { (150 AM } \\
\text { users, } 82 \text { non- } \\
\text { Users) }\end{array}$ & $\begin{array}{l}\text { Prospective } \\
\text { Cohort }\end{array}$ & Protective against thrombosis $(\mathrm{HR} 0.28,95 \% \mathrm{Cl} 0.08-0.90)$ \\
\hline $\begin{array}{l}\text { De Leeuw et al } \\
\text { [84] }\end{array}$ & 2006 & $\begin{array}{l}38 \text { SLE } H C Q \\
\text { users: } 7 \text { with } \\
\text { CVD, } 31 \text { w/o } \\
\text { CVD. }\end{array}$ & $\begin{array}{l}\text { Cross- } \\
\text { sectional }\end{array}$ & No significant change in cardiovascular riak \\
\hline Sachet et al [56] & 2007 & $\begin{array}{l}30 \text { pts: } 10 \text { SLE } \\
\text { CQ-Users, } 10 \text { SLE } \\
\text { no therapy, } 10 \\
\text { normal controls }\end{array}$ & In vivo & Improved clearance of low-density lipoproteins \\
\hline $\begin{array}{l}\text { Choojitarom e: } \\
\text { al. [85] }\end{array}$ & 2008 & $67 \mathrm{SLE}$ aPL + pts & Cohort & $\begin{array}{l}\text { Patients with lupus nephritis had higher risk of venous } \\
\text { thrombosis } O R \quad 6.2, p=0.005 \text { ) } \\
\text { Patients on } A M=\text { had decreased risk of thrombosis (OR } 0.86 \text {, } \\
p=0.02 \text { ) }\end{array}$ \\
\hline Siso et al [47]] & 2008 & $\begin{array}{l}206 \text { pts with LN } \\
\text { (56 on HCQ prev, } \\
150 \text { non-HCQ) }\end{array}$ & Cohort & $\begin{array}{l}\text { Reduced risk for hypertension ( } 32 \% \text { va, } 50 \%, p=0.027) \\
\text { Protective againat thrombosis }(5 \% \mathrm{vz}, 17 \%, p=0.039)\end{array}$ \\
\hline Kaiser et al [86] & 2009 & 1930 SLE pt: & Cohort & Protective against thrombosis (OR $0.62, p=0.0005$ ) \\
\hline $\begin{array}{l}\text { Tektonidou et al } \\
\text { [87] }\end{array}$ & 2009 & $\begin{array}{l}288 \text { pts: } 144 \text { SLE } \\
\text { aPL+ pts, } 144 \\
\text { matched SLE } \\
\text { aPL-pts }\end{array}$ & $\begin{array}{l}\text { Longitudinal } \\
\text { cohort }\end{array}$ & $\begin{array}{l}\text { Protective againgt thrombosis }- \text { duration effect } \\
\text { (aPL } \div \text { HR per month } 0.99, p=0.05 \text {; } \\
\text { aPL } \div \text { HR per month } 0.98, p=0.04 \text { ). }\end{array}$ \\
\hline Jung et al [81] & 2010 & $\begin{array}{l}54 \text { SLE pts with } \\
\text { TE (case:), } 108 \\
\text { SLE pt: w/o TE } \\
\text { (controls) }\end{array}$ & $\begin{array}{l}\text { Nested } \\
\text { case-control }\end{array}$ & Protective against thrombosis (OR $0.32,95 \% \mathrm{Cl} 0.14-0.74)$ \\
\hline Penn et al. [68] & 2010 & $\begin{array}{l}149 \text { SLE pts, } 177 \\
\text { RA pts }\end{array}$ & $\begin{array}{l}\text { Cross: } \\
\text { sectional }\end{array}$ & $\begin{array}{l}\text { Lower fasting glucose in non-diabetic women i.e. potential } \\
\text { improvement in glycaemic control } \\
\text { (SLE: } 85.9 \mathrm{vg} .89 .3 \mathrm{mg} / \mathrm{dl}, p=0.04 \text {; } \\
\text { RA: } 82.5 \mathrm{vs} .86 .6 \mathrm{mg} / \mathrm{dl}, p=0.05 \text { ) }\end{array}$ \\
\hline
\end{tabular}

Figure 2 Overview of effects of antimalarials on cardiovascular disease and thrombosis - articles from 2005-2010. CVD = cardiovascular disease. $\mathrm{aPL}=$ antiphospholipid antibodies. $\mathrm{TE}=$ thromboembolic events. $\mathrm{RA}=$ rheumatoid arthritis.

\begin{tabular}{|c|c|c|c|c|}
\hline Authors & Year & Pts & Study & Outcomes on LN \\
\hline $\begin{array}{l}\text { Trakonas et al } \\
\text { [88] }\end{array}$ & 1998 & $\begin{array}{l}47 \text { pts }(25 \\
\text { continued } \\
\text { HCQ, } 22 \\
\text { withdrew) }\end{array}$ & $\begin{array}{l}\text { Cohort } \\
\text { (randomized } \\
\text { withdrawal) }\end{array}$ & $\begin{array}{l}\text { May reduce risk and time to renal disease flare (RR 0.26; } \\
95 \% \mathrm{Cl} 0.03 \text { to } 2.54 \text { ) }\end{array}$ \\
\hline $\begin{array}{l}\text { Fesaler et al } \\
\text { LUMINA [38] }\end{array}$ & 2005 & $\begin{array}{c}518(291 \mathrm{HCQ} \\
\text { at } t=0,227 \\
\text { non-HCO) }\end{array}$ & $\begin{array}{l}\text { Longitudinal } \\
\text { observational } \\
\text { cohort }\end{array}$ & $\begin{array}{l}\text { Less likely to have renal disease ( } 8.6 \% \text { va. } 23.3 \% \text { at time }=0 \text {, } \\
\text { p:0.0001) }\end{array}$ \\
\hline $\begin{array}{l}\text { Kasitanon et al } \\
\text { (Hopkins Lupus } \\
\text { Cohort) [89] }\end{array}$ & 2006 & $\begin{array}{l}29(11 \\
\text { HCQ/MMF, } 18 \\
\text { MMF only) }\end{array}$ & Cohort & $\begin{array}{l}\text { Patients with membranous LN on MMF had improved rates } \\
\text { of renal remission within } 12 \text { months (HCQ } 7 / 11 \text { (64\%) va. } \\
\text { non-HCQ } 4 / 15 \text { (22\%); } p=0.036 \text { ) }\end{array}$ \\
\hline Sizo et al [47] & 2008 & $\begin{array}{l}206 \text { (56 pts on } \\
\text { AM prior to } \\
\text { UN diagnosis, } \\
150 \text { non-AM) }\end{array}$ & Cohort & 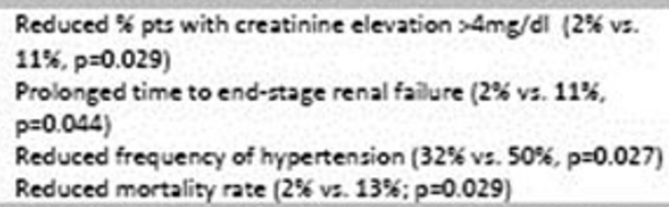 \\
\hline $\begin{array}{l}\text { Pons-Eatel et al } \\
\text { LUMINA [90] }\end{array}$ & 2009 & $\begin{array}{c}203 \text { (161 HCQ } \\
\text { users, } 42 \text { non- } \\
\text { HCQ) }\end{array}$ & $\begin{array}{l}\text { Longitudinal } \\
\text { observational } \\
\text { cohort } \\
\text { (prospective) }\end{array}$ & $\begin{array}{l}\text { Lower frequency of Class IV glomerulonephritis }(9.9 \% \mathrm{va} \text {. } \\
33.3 \% \text {, p-0.01) } \\
\text { Lower disease activity } \\
\text { Lower glucocorticoid dose (Prednisone dose } 11.3+/-12 \mathrm{mg} \\
\text { va. } 16.8+/-20.5 \mathrm{mg}, p=0.025 \text { ) } \\
\text { Protective of renal damage (full HR } 0.12,95 \% \mathrm{Cl} 0.02-0.97 \text {. } \\
p=0.0464 \text { ) } \\
\text { Lower cumulative probability of renal damage ( } p-0.0001 \text { ) }\end{array}$ \\
\hline $\begin{array}{l}\text { Shinjo et al } \\
\text { GLADEL [40] }\end{array}$ & 2010 & $\begin{array}{c}1480 \text { (1141 } \\
\text { AM users, } 339 \\
\text { non-Users) }\end{array}$ & $\begin{array}{c}\text { Observational } \\
\text { inception } \\
\text { cohort }\end{array}$ & $\begin{array}{l}\text { AM users had less renal disease prevalence }(28.4 \% \text { va. } \\
42.8 \% ; \text { p.0.001) }\end{array}$ \\
\hline
\end{tabular}

Figure 3 Overview of studies on AMs and outcomes related to LN. MMF = mycophenolate mofetil. 


\begin{tabular}{|c|c|c|}
\hline Side effect & Studies & Frequency \\
\hline \multirow[t]{5}{*}{ Retinopathy -CQ } & Leecharonen et al 2007 [8] & Retinopathy $0.18-19 \%$ \\
\hline & Marmor et al $2002[107]$ & Corneal deposits $6-7 \%$ \\
\hline & W/ang et al 1999 [103] & \\
\hline & Avina-Zubieta et al 1998 [122] & \\
\hline & Finbloom et al 1985 [123] & \\
\hline \multirow[t]{8}{*}{ Retinopathy - HCQ } & Wolfe et al 2010 [110] & Retinopathy $0-6 \%$ \\
\hline & Mavrikakis et al 2003 [108] & Corneal deposits $0.8 \%$ \\
\hline & Marmor et al 2002 [107] & \\
\hline & W/anget al 1999[103] & \\
\hline & Avina-Zubieta 1998 [122] & \\
\hline & Levyet al 1997 [111] & \\
\hline & Spalton et al 1993 [124] & \\
\hline & Finbloom et al 1985 [123] & \\
\hline Ototoxicity & $\begin{array}{l}\text { Wang et al } 1999 \text { [103] } \\
\text { Morand et al } 1992 \text { [125] }\end{array}$ & Ototoxicity $0-0.6 \%$ \\
\hline \multirow[t]{4}{*}{ Cardiotoxicity } & $\begin{array}{l}\text { Costedoat-Chalumeau et al } \\
2007[113,126]\end{array}$ & $\begin{array}{l}\text { Heart conduction defect } 0-4 \% \\
\text { myocardiopathy and AV block }<1 \%\end{array}$ \\
\hline & Wozniacka et al 2006[127] & Case report: of cardiomyopathy, heart conduction \\
\hline & Nord et al 2004 [112] & disturbances, congestive heart failure \\
\hline & Cervera et al 2001 [128] & \\
\hline \multirow[t]{6}{*}{ Cutaneous } & Kalia et al 2010 [6] & Skin rash $0.6-4.3 \%$ \\
\hline & Di Giacomo et al. 2009 [115] & Hyperpigmentation - 10 to $30 \%$ (quinacrines $\mathrm{CO} / \mathrm{HCO}$ ) \\
\hline & Puri et al 2008 [114] & Urticaria $12 \%$ \\
\hline & Herman et al 2006 [118] & Psoriasis - insufficient evidence to suggest flare \\
\hline & Wang et al 1999 [103] & causality \\
\hline & $\begin{array}{c}\text { Avina-Zubieta et al } 1998 \text { [122] } \\
\text { Morand et al } 1992 \text { (125] }\end{array}$ & \\
\hline \multirow[t]{5}{*}{ Gastrointestinal } & Bezerra et al 2005 [129] & Gastrointestinal $0-30 \%$ \\
\hline & Van Beck, Piette 2001 & Nausea / vomiting $12 \%$ \\
\hline & W/ang et al 1999 [103] & Diarrhoea $18 \%$ \\
\hline & Avina-Zubieta et al 1998 [122] & Elevated LFT: $-10 \%$ \\
\hline & Morand et al 1992 [125] & \\
\hline \multirow[t]{5}{*}{ Other } & Casado et al 2006 [119] & Headachea $1.3-12 \%$ \\
\hline & Bezerra et al 2005 [129] & Myopathy 0-6.7\% \\
\hline & Wang et al 1999 [103] & \\
\hline & Avina-Zubieta et al 1998 [122] & \\
\hline & Morand et al 1992 [125] & \\
\hline \multirow[t]{4}{*}{ Rare (case reports) } & Kalia et al 2010 [6] & Haemolyais in G6PD deficiency patients. \\
\hline & & Severe leucopenia, aplastic anaemia \\
\hline & Collins et al 2008 [120] & Acute psychosis from CQ (PCP-like) \\
\hline & Bracamonte et al 2006 [121] & Pseudo-fabry disease \\
\hline
\end{tabular}

used in SLE, however less known is its role in lupus nephritis. Our study examined the history of AM use, theorized mechanisms of action, efficacy in SLE, in particular in lupus nephritis, safety in pregnancy, and overall safety profile.

\section{Methods}

We conducted a search of all relevant literature using Medline (OVID and EMBASE) and PubMed. We included randomized-controlled trials, observational cohort studies, and case-control studies. Case reports were only included for the adverse effect profile of AM.

\section{Results}

-AM use benefits patients with SLE including improving survival, reducing disease activity, new organ involvement, integument damage, risk of infection, risk of 
thrombosis, and possible cardioprotective and antimalignancy effects.

-In lupus nephritis, AM use improves time to endstage renal disease, disease activity, flare rates, disease remission as an adjunct with other immunomodifying drugs, and reduced cumulative corticosteroid use.

-AM are safe to use and should be continued in pregnant SLE patients for its beneficial effects of reducing disease activity, flare rates, cumulative corticosteroid requirements, and possible reduction in development of cardiac neonatal lupus erythematosus. AM have a good safety profile, with gastrointestinal symptoms being the most common. Careful regular monitoring for retinopathy is recommended as per American Academy of Ophthalmology.

-In patients with renal disease, caution with dosing and careful monitoring for adverse events should be taken.

\section{Conclusion}

AM are medications which confer many benefits to patients with SLE and lupus nephritis, with a good safety profile.

\section{Disclosure}

Senq-J. Lee: None; Earl D. Silverman: None; Joanne M. Bargman: None.

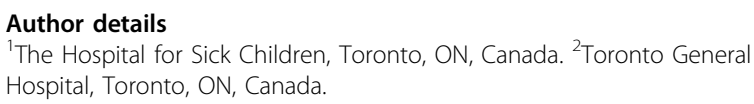

Published: 13 July 2012
Submit your next manuscript to BioMed Central and take full advantage of:

- Convenient online submission

- Thorough peer review

- No space constraints or color figure charges

- Immediate publication on acceptance

- Inclusion in PubMed, CAS, Scopus and Google Scholar

- Research which is freely available for redistribution

Submit your manuscript at www.biomedcentral.com/submit 